\title{
STRUCTURE THEORY FOR A CLASS OF GRADE FOUR GORENSTEIN IDEALS
}

\author{
BY \\ ANDREW KUSTIN $^{1}$ AND MATTHEW MILLER ${ }^{2}$
}

\begin{abstract}
An ideal $I$ in a commutative noetherian ring $R$ is a Gorenstein ideal of grade $g$ if $\operatorname{pd}_{R}(R / I)=$ grade $I=g$ and the canonical module $\operatorname{Ext}_{R}^{8}(R / I, R)$ is cyclic. Serre showed that if $g=2$ then $I$ is a complete intersection, and Buchsbaum and Eisenbud proved a structure theorem for the case $g=3$. We present generic resolutions for a class of Gorenstein ideals of grade 4, and we illustrate the structure of the resolution with various specializations. Among these examples there are Gorenstein ideals of grade 4 in $k[[x, y, z, v]]$ that are $n$-generated for any odd integer $n>7$. We construct other examples from almost complete intersections of grade 3 and their canonical modules. In the generic case the ideals are shown to be normal primes. Finally, we conclude by giving an explicit associative algebra structure for the resolutions. It is this algebra structure that we use to classify the different Gorenstein ideals of grade 4, and which may be the key to a complete structure theorem.
\end{abstract}

Introduction. One of the most useful techniques in the study of ideals of finite projective dimension has been to realize such ideals as specializations of generic ideals whose structure and resolutions are well understood. Of these the various determinantal ideals play a preeminent role, and in many cases explicit resolutions have been constructed. Moreover, for perfect ideals the structure of the resolution is preserved under specialization so long as the grade of the ideal is maintained.

In $\$ 2$ we present generic resolutions for a class (defined below) of Gorenstein ideals of grade four. As a corollary we show that there are Gorenstein ideals of grade four in local rings that are minimally generated by any number $n$ elements, where $n \geqslant 6$. We exploit the structure of the resolution to prove a regularity result, which shows that the generic ideals are normal primes. In $\$ 3$ we present a construction that produces examples in our class. Finding the generic resolution represents the first step toward a description and classification of all Gorenstein ideals of grade four. The next step is to show that any ideal in the class is indeed a specialization of the generic case.

A similar program has been carried out successfully for Gorenstein local rings $R / I$ of codimension three by Buchsbaum and Eisenbud [4]. To accomplish this they exploited the algebra structure on a minimal free resolution of $R / I$. They

Received by the editors July 31, 1980 and, in revised form, February 17, 1981. Some of the contents of this paper were presented to the special session on commutative algebra at the American Mathematical Society Meeting in Philadelphia on April 18, 1980.

1980 Mathematics Subject Classification. Primary 13C05; Secondary: 13D25, 13H10, 14M12, 16 A03.

${ }^{1}$ Research supported by grant \#3093-X0-0038 of the University of Kansas General Research Allocation.

${ }^{2}$ Research supported by a grant from the University of Tennessee. 
found that there is essentially just one kind of resolution: the ideal $I$ is presented by an alternating matrix and is generated by the maximal pfaffians of this matrix.

The situation is more complicated in codimension four. In [17] we proved that if $(R, \mathrm{~m}, k)$ is a local Gorenstein ring in which 2 is a unit, then a minimal resolution of $R / I$ admits an associative algebra structure. In $\$ 4$ we give an explicit algebra structure on the resolutions discussed in $\$ 2$, and we are able to drop the restrictive hypotheses on $R$. The induced algebra structure on $\Lambda_{0}=\operatorname{Tor}_{.}^{R}(R / I, k)$ agrees with the usual multiplication on the homology algebra. In codimension three, with the exception of the complete intersections, one always has $\Lambda_{1}^{2}=0$, but in codimension four we have found $\operatorname{dim}_{k} \Lambda_{1}^{2}$ to be a useful invariant in distinguishing resolutions of different forms. All known examples fall into one of the following classes.

(i) $\Lambda_{1}^{2}=\Lambda_{2}$ if and only if $I$ is a complete intersection.

(ii) $\operatorname{dim} \Lambda_{1}^{2}=(1 / 2) \operatorname{dim} \Lambda_{2}$. This class includes the hypersurface sections of a local Gorenstein ring of codimension three.

(iii) $\operatorname{dim} \Lambda_{1}^{2}=3$. In Theorem 2.3 we present a family of generic ideals in this class. Example 3.7 exhibits a family of specializations that are produced from perfect almost complete intersections of grade three and their canonical modules.

(iv) $\Lambda_{1}^{2}=0$. This class includes the ideals generated by the $(n-1) \times(n-1)$ minors of an $n \times n$ matrix, see Herzog [9].

In [18] we showed that there are no ideals with (1/2)dim $\Lambda_{2}<\operatorname{dim} \Lambda_{1}^{2}<\operatorname{dim} \Lambda_{2}$ and that there is good reason to believe the hypersurface section examples actually exhaust class (ii).

There is strong evidence that control of the algebra structure will enable us to show that any ideal in class (iii) is a specialization of the generic case. We state a result that completes this step for the "smallest" ideals in class (iii), namely the 7-generated ones, but as the result is incomplete, we omit the proof.

1. Preliminaries. We begin by establishing the notation needed in the manipulation of pfaffians. Let $Y$ be an $n \times n$ alternating matrix $\left(y_{i j}=-y_{j i}\right.$ and $\left.y_{i i}=0\right)$ with entries in a commutative ring $R$. If $r<n$ we define $\operatorname{Pf}_{i_{1}} \ldots, i_{r}(Y)$ to be the pfaffian of the alternating submatrix of $Y$ formed by deleting rows and columns $i_{1}, \ldots, i_{r}$. (The determinant of such a matrix is a perfect square in $R$; the pfaffian is a uniquely determined square root. See Lang [19, p. 372] or Artin [1, p. 140].) Let (i) denote the multi-index $i_{1} i_{2} \ldots i_{r}$. Define $\sigma(i)$ to be 0 if $(i)$ has a repeated index and otherwise to be the sign of the permutation that rearranges $i_{1}, \ldots, i_{r}$ in ascending order. Let $|i|=\sum_{\nu=1}^{r} i_{\nu}$ and let

$$
Y_{(i)}=(-1)^{|i|+1} \sigma(i) \operatorname{Pf}_{(i)}(Y) .
$$

If $r=n$ let $Y_{(i)}=(-1)^{|i|+1} \sigma(i)$, and if $r>n$ let $\gamma_{(i)}=0$. Finally, let $\mathbf{y}=$ $\left[Y_{1}, \ldots, Y_{n}\right]$ be the vector of pfaffians of $Y$ of order $n-1$, signed appropriately according to the conventions described above. There is a "Laplace expansion" for developing pfaffians in terms of ones of lower order.

LEMMA 1.1. Let $Y$ be an $n \times n$ alternating matrix and $j$ a fixed integer, $1 \leqslant j \leqslant n$. Then

(a) $\operatorname{Pf}(Y)=\sum_{i=1}^{n} y_{i j} Y_{i j}$, and

(b) $\mathbf{y} Y=0$. 
Proof. The first assertion is an exercise in Artin [1, p. 142]; the second follows by applying the first to an augmented matrix formed by repeating a row and corresponding column of $Y$. The next result also follows from (a).

LEMMA 1.2. Let $Y$ be an $n \times n$ alternating matrix. Suppose that $a, b, c, d$, and $l$ are in $\{1, \ldots, n\}$ with $a, b, c, d$ distinct. Then

$$
\sum_{k=1}^{n} y_{k l} Y_{k a b}=-\delta_{l a} Y_{b}+\delta_{l b} Y_{a}
$$

and

$$
\sum_{k=1}^{n} y_{k l} Y_{k a b c d}=-\delta_{l a} Y_{b c d}+\delta_{l b} Y_{a c d}-\delta_{l c} Y_{a b d}+\delta_{l d} Y_{a b c} .
$$

LEMmA 1.3. Let $B$ and $Z$ be $3 \times 3$ alternating matrices. Then $\mathrm{b} Z+\mathrm{z} B=0$.

Proof. A direct computation establishes the result.

The following example illustrates the expansion formula, and will be used in the sequel.

EXAMPLE 1.4. Let $\tau \geqslant 3$ be an odd integer and let $\lambda=(\tau+1) / 2$. Let $Y$ be the $\tau \times \tau$ alternating matrix with entries

$$
y_{i j}= \begin{cases}x & \text { if } i \text { is odd and } j=i+1, \\ y & \text { if } i \text { is even and } j=i+1, \\ z & \text { if } j=\tau-i+1\end{cases}
$$

for $i<j$. This is the matrix $H_{\tau}$ of Buchsbaum and Eisenbud [4, Proposition 6.2]. Now by Lemma 1.1(a)

$$
\begin{aligned}
Y_{1} & =\operatorname{Pf}_{1}(Y)= \pm y_{\tau \tau-1} \operatorname{Pf}_{1 \tau-1 \tau}(Y) \\
& = \pm y_{\tau \tau-1} y_{23} \operatorname{Pf}_{123 \tau-1 \tau}(Y) \\
& \vdots \\
& = \pm y_{\tau \tau-1} y_{23} y_{\tau-2 \tau-3} y_{45} y_{\tau-4 \tau-5} \cdots \\
& = \pm y^{\lambda-1}
\end{aligned}
$$

and similarly $Y_{\tau}= \pm x^{\lambda-1}$. To compute $Y_{\lambda}$ we use Lemma 1.1(a) (always expanding across the top row); it turns out that $Y_{\lambda}=z^{\lambda-1}+p$ where $p$ is in $(x, y)$.

Let $R$ be a commutative noetherian ring and let $I$ be a proper ideal of $R$ with finite projective dimension. The grade of $I$ is the length of a maximal $R$-sequence contained in $I$. (If $R$ is Cohen-Macaulay, then the grade of $I$ equals the height of $I$. If $R$ is also local, then both coincide with the (embedding) codimension of $R / I$.) The ideal $I$ is called perfect if grade $I=\operatorname{pd}_{R} R / I$. An ideal $I$ of grade $g$ is called a Gorenstein ideal if $I$ is perfect and $\operatorname{Ext}_{R}^{g}(R / I, R)$ is a cyclic $R / I$-module. It follows from Bass [2, Proposition 5.1] that if $I$ is a Gorenstein ideal of a Gorenstein ring $R$, then $R / I$ is also a Gorenstein ring.

Let us further assume that $R$ is a local ring with maximal ideal $m$ and residue field $k$. Let $\mathbf{F}: 0 \rightarrow F_{g} \rightarrow \cdots \rightarrow F_{2} \rightarrow^{d_{2}} F_{1} \rightarrow^{d_{1}} R$ be a minimal free resolution of $R / I$ and let $\Lambda_{\mathrm{r}}=\operatorname{Tor}^{R}(R / I, k)=\mathrm{F} \otimes k$. If $I$ is perfect, then the type of $I$ is 
$\operatorname{dim}_{k} \Lambda_{g}$, or equivalently, rank $F_{g}$. (We note that $I$ has type 1 if and only if $\operatorname{Ext}_{R}^{g}(R / I, R)$ is cyclic.) The minimal number of generators of $I$ is denoted $\mu(I)$.

Any comparison map from the complex $F \otimes_{R} F$ to the resolution $F$ which covers the multiplication map $(R / I) \otimes_{R}(R / I) \rightarrow R / I$ induces the usual algebra on the homology $\operatorname{Tor}^{R}(R / I, k)$. (See Cartan and Eilenberg [6, Chapter XI, §\$1-4].) Let $C$ be the image of $d_{2}$ in $F_{1}$ and let $K$ be the $R$-submodule of $C$ generated by the Koszul relations on the entries of $d_{1}$. The map $d_{2}: F_{2} \rightarrow C$ is a minimal surjection, and $d_{2} \otimes 1$ carries $\Lambda_{1}^{2}$ onto $K \otimes k$. Consequently

$$
\operatorname{dim}_{k} \Lambda_{1}^{2}=\operatorname{dim}_{k}(K \otimes k)=\operatorname{dim}_{k}(K+\mathfrak{m} C) / \mathfrak{m} C .
$$

In other words $\operatorname{dim} \Lambda_{1}^{2}$ is just the maximum number of Koszul relations that can occur as columns in a matrix for $d_{2}$.

2. The generic resolution. When we began our investigation there were few examples of grade four Gorenstein ideals with explicitly given generators or resolutions. Hypersurface sections of grade three Gorenstein ideals are well known. So are the ideals generated by the $(n-1) \times(n-1)$ minors of an $n \times n$ matrix; for example see Gulliksen and Negard [7]. Various scattered examples have appeared in the literature: Hochster and Roberts [13, Example 2.2], Stanley [23, Example 8.4], Herzog [8, §4]. There is an unpublished example due to Fossum: the ideal of $2 \times 2$ minors of a $2 \times 2 \times 2$ array. Other examples arise from coordinate rings of curves in 5-space parametrized by symmetric numerical semigroups; see, for example, Herzog and Kunz [11], K. Watanabe [25], and Bresinsky [3]. Each of these examples falls into one of the classes described in the introduction. The hypersurface sections have $\operatorname{dim} \Lambda_{1}^{2}=(1 / 2) \operatorname{dim} \Lambda_{2}$. Herzog's vector-matrix example and some of the symmetric semigroup curves have $\operatorname{dim} \Lambda_{1}^{2}=3$. All the other cited examples have $\Lambda_{1}^{2}=0$.

In this paper we describe a method (Lemma 3.2) for constructing grade four Gorenstein ideals from perfect ideals of grade three and their canonical modules. Using this method we can produce examples belonging to each of the latter three classes of $\operatorname{dim} \Lambda_{1}^{2}$. But to fully utilize the method of Lemma 3.2, we must have a well-studied class of perfect grade three ideals. The class of almost complete intersections fits this bill. In Example 3.7 we show that $I+v J$ is a grade four Gorenstein ideal provided $I$ and $J$ are geometrically linked ideals in general position with $I$ a grade three almost complete intersection, $J$ a grade three Gorenstein ideal, and $v$ a regular element on $R / I$.

Each of the ideals $I+v J$ constructed in Example 3.7 is in the class $\operatorname{dim} \Lambda_{1}^{2}=3$. Unfortunately these ideals do not exhaust the class, as we illustrate by recording the free resolution of $R / \mathfrak{A}$ for $\mathfrak{A}$ the most general 7-generated ideal of the form $I+v J$. Let $v$ be an indeterminate, $A$ be a $3 \times 3$ matrix of indeterminates, and $\mathbf{y}$ be a $1 \times 3$ matrix of indeterminates. Let $R$ be the polynomial ring obtained by adjoining these indeterminates to the integers; i.e. $R=\mathbf{Z}[A, \mathbf{y}, v]$. Let $\mathbf{z}$ be the $1 \times 3$ matrix $-\mathbf{y} A$,

$$
Y=\left[\begin{array}{rrr}
0 & Y_{3} & -Y_{2} \\
-Y_{3} & 0 & Y_{1} \\
Y_{2} & -Y_{1} & 0
\end{array}\right] \text { and } Z=\left[\begin{array}{rrr}
0 & Z_{3} & -Z_{2} \\
-Z_{3} & 0 & Z_{1} \\
Z_{2} & -Z_{1} & 0
\end{array}\right]
$$


The minimal resolution of $R / \mathfrak{A}$ is given by

$$
0 \rightarrow R \stackrel{d_{4}}{\rightarrow} R^{7} \stackrel{d_{3}}{\rightarrow} R^{12} \stackrel{d_{2}}{\rightarrow} R^{7} \stackrel{d_{1}}{\rightarrow} R
$$

with $d_{1}=[\mathbf{z}|v \mathbf{y}|-\operatorname{det} A], d_{4}=d_{1}^{t}$,

$$
d_{2}=\left[\begin{array}{cccc}
A & -\operatorname{Adj}(A) & v I & 0 \\
0 & 0 & A & Y \\
0 & \mathbf{y} & 0 & 0
\end{array}\right]
$$

and

$$
d_{3}=\left[\begin{array}{ll}
0 & I \\
I & 0
\end{array}\right] d_{2}^{t}
$$

No specialization of this resolution yields the resolution associated to a vector and a matrix as described by Herzog [8]. His resolution is isomorphic to one that has the same block decomposition as (1), but the indeterminates play new roles. In this case

$$
\begin{aligned}
& d_{1}=[\mathbf{z}|\mathbf{b} \operatorname{Adj}(A)|-\operatorname{det} A], \\
& d_{2}=\left[\begin{array}{cccc}
Z & -\operatorname{Adj}(A) & 0 & -B A^{t} \\
0 & u I & A & Y \\
0 & \mathbf{y} & \mathbf{b} & 0
\end{array}\right] .
\end{aligned}
$$

Here b is a $1 \times 3$ matrix of indeterminates, $u$ is an indeterminate, $R=\mathbf{Z}[\mathbf{y}, A, \mathbf{b}, u]$, $\mathbf{z}=u \mathbf{b}-\mathbf{y} A$, and

$$
B=\left[\begin{array}{rrr}
0 & b_{3} & -b_{2} \\
-b_{3} & 0 & b_{1} \\
b_{2} & -b_{1} & 0
\end{array}\right]
$$

We seek therefore a resolution sufficiently general that both (1) and (2) can be realized as specializations. The resolution given by

$$
\begin{aligned}
& d_{1}=[\mathbf{z}|v \mathbf{y}+\mathbf{b} \operatorname{Adj}(A)|-\operatorname{det} A-u v], \\
& d_{2}=\left[\begin{array}{cccc}
Z & -\operatorname{Adj}(A) & v I & -B A^{t} \\
0 & u I & A & Y \\
0 & \mathbf{y} & \mathbf{b} & 0
\end{array}\right], \\
& d_{3}=\left[\begin{array}{ll}
0 & I \\
I & 0
\end{array}\right] d_{2}^{t}, \quad d_{4}=d_{1}^{t}
\end{aligned}
$$

over $R=\mathbf{Z}[\mathbf{y}, A, \mathbf{b}, u, v]$, with $\mathbf{z}$ and $B$ as in (2), accomplishes just what we want. It specializes to (1) or (2) by setting $u$ and $b$ or $v$ respectively to zero. In fact (3) is the smallest example of the generic resolution given in Theorem 2.3. We have chosen to represent the most general resolution first and to exhibit its specializations (especially the $I+v J$ examples) in $\$ 3$. 
Before constructing the complex we fix the notation. The following are given:

$$
\begin{array}{ll}
\tau \geqslant 3, & \text { odd integer, } \\
Y, & \tau \times \tau \text { alternating matrix, } \\
X, & (\tau+1) \times 3 \text { matrix, } \\
A, & \text { upper } \tau \times 3 \text { submatrix of } X, \\
\text { b, } & \tau+1 \text { row of } X, \\
u, v, & \text { scalars. }
\end{array}
$$

The following are to be defined:

$$
\begin{array}{ll}
w, & \text { scalar, } \\
S, & 3 \times \tau \text { matrix, } \\
T, & 3 \times \tau \text { matrix, } \\
Z, & 3 \times 3 \text { alternating matrix. }
\end{array}
$$

Let $d_{i j k}$ be the determinant of the $3 \times 3$ submatrix of $X$ formed by rows $i, j, k$ (in this order), and let

$$
w=\sum_{1<i<j<k<\tau} d_{i j k} Y_{i j k} .
$$

Define $Z$ to be the $3 \times 3$ alternating matrix whose pfaffian row vector is

$$
\mathbf{z}=u \mathbf{b}-\mathbf{y} A \text {. }
$$

That is

$$
Z=\left[\begin{array}{rrr}
0 & Z_{3} & -Z_{2} \\
-Z_{3} & 0 & Z_{1} \\
Z_{2} & -Z_{1} & 0
\end{array}\right]
$$

where $Z_{i}=u x_{\tau+1, i}-\sum_{j=1}^{\tau} Y_{j} x_{j i}$. Let $S$ be the $3 \times \tau$ matrix with entries

$$
s_{l k}=(-1)^{l+1} \sum_{1<i<j<\tau} Y_{k i j}\left|\begin{array}{ll}
x_{i m} & x_{i n} \\
x_{j m} & x_{j n}
\end{array}\right|
$$

where $m<n$ and $\{l, m, n\}=\{1,2,3\}$. Let $B$ be the $3 \times 3$ alternating matrix whose pfaffian row vector is $\mathbf{b}$. Then let $T$ be the $3 \times \tau$ matrix $-B A^{t}$.

We are now ready to construct the complex. The maps are defined as follows.

$$
\begin{aligned}
& d_{1}=[\mathbf{z}|v \mathbf{y}-\mathbf{b} S| w-u v], \\
& d_{2}=\left[\begin{array}{c|c|c|c}
Z & S & v I & T \\
\hline 0 & u I & & Y \\
\hline 0 & \mathbf{y} & X & 0
\end{array}\right], \\
& d_{3}=\left[\begin{array}{cc}
0 & I_{\tau+3} \\
I_{\tau+3} & 0
\end{array}\right] d_{2}^{t}, \\
& d_{4}=d_{1}^{t} .
\end{aligned}
$$

THEOREM 2.1. With the maps defined above

$$
\text { F: } 0 \rightarrow R \stackrel{d_{4}}{\rightarrow} R^{4+\tau} \stackrel{d_{3}}{\rightarrow} R^{2(\tau+3)} \stackrel{d_{2}}{\rightarrow} R^{4+\tau} \stackrel{d_{1}}{\rightarrow} R
$$

is a complex. 
The proof relies on three matrix identities.

\section{LEMMA 2.2.}

(a) $S A=w I$,

(b) $S Y=(Z-u B) A^{t}$,

(c) $w \mathbf{y}=\mathbf{y} A S$.

\section{ProOF.}

(a)

$$
\begin{aligned}
& (S A)_{l p}=\sum_{k=1}^{\tau} s_{l k} x_{k p}=\sum_{k=1}^{\tau}(-1)^{l+1} \sum_{1<i<j<\tau} Y_{k i j}\left|\begin{array}{ll}
x_{i m} & x_{i n} \\
x_{j m} & x_{j n}
\end{array}\right| x_{k p} \\
& =(-1)^{l+1} \sum_{1<\alpha<\beta<\gamma<\tau} Y_{\alpha \beta \gamma}\left\{x_{\alpha p}\left|\begin{array}{ll}
x_{\beta m} & x_{\beta n} \\
x_{\gamma m} & x_{\gamma n}
\end{array}\right|\right. \\
& \left.-x_{\beta p}\left|\begin{array}{cc}
x_{\alpha m} & x_{\alpha n} \\
x_{\gamma m} & x_{\gamma n}
\end{array}\right|+x_{\gamma p}\left|\begin{array}{ll}
x_{\alpha m} & x_{\alpha n} \\
x_{\beta m} & x_{\beta n}
\end{array}\right|\right\} \\
& =Y_{\alpha \beta \gamma} \delta_{l p} d_{\alpha \beta \gamma}=(w I)_{p} . \\
& (S Y)_{l p}=\sum_{k=1}^{\tau} s_{l k} y_{k p}=\sum_{k}(-1)^{l+1} \sum_{1<i<j<\tau} Y_{k i j}\left|\begin{array}{ll}
x_{i m} & x_{i n} \\
x_{j m} & x_{j n}
\end{array}\right| y_{k p} \\
& =(-1)^{l+1} \sum_{i<j}\left(\sum_{k} y_{k p} Y_{k i j}\right)\left|\begin{array}{ll}
x_{i m} & x_{i n} \\
x_{j m} & x_{j n}
\end{array}\right| \\
& =(-1)^{l+1}\left\{\sum_{j>p=i}\left(-Y_{j}\right)\left|\begin{array}{ll}
x_{p m} & x_{p n} \\
x_{j m} & x_{j n}
\end{array}\right|+\sum_{i<p=j} Y_{i}\left|\begin{array}{ll}
x_{i m} & x_{i n} \\
x_{p m} & x_{p n}
\end{array}\right|\right\} \\
& \text { by Lemma } 1.2 \\
& =(-1)^{l} \sum_{q=1}^{\tau} Y_{q}\left|\begin{array}{ll}
x_{p m} & x_{p n} \\
x_{q m} & x_{q n}
\end{array}\right| .
\end{aligned}
$$

On the other hand we have

$$
\left((u B-Z) A^{t}\right)_{l p}=(u B-Z)_{l m} x_{p m}+(u B-Z)_{l n} x_{p n}
$$

where $\{l, m, n\}=\{1,2,3\}$ and $m<n$. Now by equation (5) the right-hand side is

(c)

$$
\begin{aligned}
(-1)^{l+1}\left\{(\mathrm{y} A)_{n} x_{p m}-(\mathbf{y} A)_{m} x_{p n}\right\}=(-1)^{l+1} \sum_{q=1}^{\tau} Y_{q}\left|\begin{array}{ll}
x_{p m} & x_{p n} \\
x_{q m} & x_{q n}
\end{array}\right|=-(S Y)_{\varphi} . \\
(A S)_{p k}=\sum_{l=1}^{3} x_{p l} s_{l k}=\sum_{l} x_{p l} \sum_{1<i<j<\tau}(-1)^{l+1} Y_{k i j}\left|\begin{array}{ll}
x_{i m} & x_{i n} \\
x_{j m} & x_{j n}
\end{array}\right| \\
=\sum_{i<j} Y_{k i j} d_{p i j} .
\end{aligned}
$$

Recall that $w=\sum Y_{\alpha \beta \gamma} d_{\alpha \beta \gamma}$ where $1 \leqslant \alpha<\beta<\gamma \leqslant \tau$. Hence

$$
(w I-A S)_{p k}=\sum_{\alpha<\beta<\gamma} d_{\alpha \beta \gamma}\left[Y_{\alpha \beta \gamma} \delta_{p k}-Y_{k \beta \gamma} \delta_{p \alpha}+Y_{k \alpha \gamma} \delta_{p \beta}-Y_{k \alpha \beta} \delta_{p \gamma}\right]
$$

and by Lemma 1.2 the right-hand side is

$$
\sum_{\alpha<\beta<\gamma} d_{\alpha \beta \gamma} \sum_{j=1}^{\tau} y_{j p} Y_{j \alpha \beta \gamma k}=\sum_{j=1}^{\tau} y_{p j}\left(\sum_{\alpha<\beta<\gamma}-d_{\alpha \beta \gamma} Y_{j \alpha \beta \gamma k}\right) .
$$


If we take $N$ to be the matrix with entries $n_{j k}=\Sigma_{\alpha<\beta<\gamma}-d_{\alpha \beta \gamma} Y_{j \alpha \beta \gamma k}$ then

$$
w I-A S=Y N
$$

and by Lemma 1.1(b), $\mathbf{y}(w I-A S)=0$.

We now turn to the proof of Theorem 2.1.

Claim. $d_{1} d_{2}=0$ and $d_{3} d_{4}=0$. We have $\mathbf{z} Z=0$ by Lemma 1.1. Using Lemma 2.1(a) and (c) and equation (5), we find $\mathbf{z} S+v u \mathbf{y}-u \mathbf{b} S+(w-v u) \mathbf{y}=0$ and also $v \mathbf{z}+v \mathbf{y} A-\mathbf{b} S A+(w-v u) \mathbf{b}=0$. Using $T=-B A^{t}$ and Lemmas 1.1, 1.3, and 2.1(b), we obtain

$$
\mathbf{z} T+v \mathbf{y} Y-\mathbf{b} S Y=0 .
$$

The second assertion follows immediately.

Claim. $d_{2} d_{3}=0$. First we note that

$$
d_{3}=\left[\begin{array}{ll}
0 & I \\
I & 0
\end{array}\right] d_{2}^{t}=\left[\begin{array}{ccc}
v I & A^{t} & \mathbf{b}^{t} \\
T^{t} & -Y & 0 \\
-Z & 0 & 0 \\
S^{t} & u I & \mathbf{y}^{t}
\end{array}\right]
$$

since $Y$ and $Z$ are alternating. By Lemma 2.2 we have $v Z+S T^{t}-v Z+T S^{t}=$ $-w\left(B+B^{t}\right)=0$ and $Z A^{t}-S Y+u T=0$. Finally, using Lemmas 1.1 and 1.3 in transposed form, we find

$$
Z \mathbf{b}^{t}+T \mathbf{y}^{t}=Z \mathbf{b}^{t}+u B \mathbf{b}^{t}-B A^{t} \mathbf{y}^{t}=Z \mathbf{b}^{t}+B \mathbf{z}^{t}=0
$$

The rest of the details present no new problems.

Let $\mathfrak{A}=\operatorname{im}\left(d_{1}\right)$. We claim that $F$ is a resolution of $R / \mathfrak{A}$ if grade $\mathfrak{A}=4$. To show this we demonstrate that $\mathbf{F}$ is "generically exact". Let $R=\mathbf{Z}\left[x_{i j}, y_{l k}, u, v\right]$ be the polynomial ring in indeterminates $x_{i j}$ (for $1 \leqslant i \leqslant \tau+1$ and $1 \leqslant j \leqslant 3$ ), $y_{l k}=-y_{k l}$ (for $1 \leqslant l<k \leqslant \tau$ ), $u$, and $v$. Let $\mathbf{G}$ denote the complex (6).

THEOREM 2.3. The generic complex $\mathbf{G}$ is a resolution of $R / \mathfrak{A}$.

Proof. We reduce the problem to showing that a certain specialization is exact; this simplifies the grade computations. Let $Y$ be the matrix $H_{\tau}$ of Example 1.4. Set all $x_{i j}=0$ except for $x_{11}=y, x_{\lambda 2}=z$, and $x_{\tau 3}=x$. Consequently $\mathbf{b}=0$ and $T=0$. In effect we pass to a factor ring $R^{\prime}=R / p$. Let $\mathbf{G}^{\prime}=\mathbf{G} \otimes R^{\prime}$. In Lemma 2.4 we prove that $\mathbf{G}^{\prime}$ is exact. Assuming this, we apply the Buchsbaum-Eisenbud exactness criterion [5] to verify that $\mathbf{G}$ is exact. Since $\mathbf{G}^{\prime}$ is exact the maps $d_{i}^{\prime}$ have the expected ranks $r_{i}$; here $r_{1}=r_{4}=1$ and $r_{2}=r_{3}=\tau+3$. Exactness of $\mathbf{G}^{\prime}$ further implies that grade $\left(I_{r_{i}}\left(d_{i}^{\prime}\right)\right) \geqslant i$ for $i=1, \ldots, 4$. Since $R$ and $R^{\prime}$ are regular, grade may be replaced by height. If $I=I_{r_{i}}\left(d_{i}\right)$ and $I^{\prime}=I_{r_{i}}\left(d_{i}^{\prime}\right)$, then

$$
I^{\prime}=(I+\mathfrak{p}) / \mathfrak{p}
$$

If we assign the variables $u$ and $v$ the weights $\operatorname{deg} v=2$ and $\operatorname{deg} u=(\tau-1) / 2$, then each ideal $I_{r_{i}}\left(d_{i}\right)$ is homogeneous. Consequently $\mathfrak{p}+\mathfrak{q}$ is proper for any associated prime $q$ of $I$, and by Serre's altitude formula [22, V-18] we conclude $\mathrm{ht}(I+\mathfrak{p}) \leqslant \mathrm{ht} I+\mathrm{ht} \mathfrak{p}$. On the other hand, since $R$ is catenary ht ${ }_{R^{\prime}} I^{\prime}=\mathrm{ht}(I+\mathfrak{p})$ - ht $\mathfrak{p}$. Hence $\operatorname{grade}\left(I_{r_{i}}\left(d_{i}\right)\right)=\mathrm{ht} I \geqslant i$ for each $i$. In particular $I_{r_{i}}\left(d_{i}\right) \neq 0$ so 
$\operatorname{rank}\left(d_{i}\right) \geqslant r_{i}$. Clearly, however, rank $d_{1}=\operatorname{rank} d_{4}=1$. If rank $d_{3}>\tau+3$ then since $R$ is a domain $d_{3}$ would be injective, which is impossible since $\operatorname{im}\left(d_{4}\right) \neq 0$. If rank $d_{2}>\tau+3$ then $\operatorname{im}\left(d_{2}\right)_{(0)}=R_{(0)}^{\tau+4}$ and $\left(d_{1}\right)_{(0)}=0$, again a contradiction.

LEMMA 2.4. The specialization $\mathbf{G}^{\prime}$ is exact.

Proof. The $(\tau+3) \times(\tau+3)$ submatrix of $d_{2}$ formed by omitting row 1 and columns 1,5 through $\tau+3$, and $\tau+5$ through $\tau+7$ has determinant $\pm y^{3} Y_{1}^{5}$. Similarly $\pm x^{3} Y_{\tau}^{5}$ and $\pm z^{3} Y_{\lambda}^{5}$ are $(\tau+3) \times(\tau+3)$ minors of $d_{2}^{\prime}$. By Example 1.4, $(x, y, z)$ is contained in $\operatorname{rad}\left(I_{\tau+3}\left(d_{2}^{\prime}\right)\right)$. Thus grade $\left(I_{\tau+3}\left(d_{2}^{\prime}\right)\right) \geqslant 3$. Since $d_{3}^{\prime}$ has the same minors as $d_{2}^{\prime}$, the rank and grade criteria hold for both maps. (The same arguments as used above demonstrate that the ranks are not too large.) Now $\mathfrak{A}=\operatorname{im}\left(d_{1}\right)$ is the ideal $\left(y Y_{1}, z Y_{\lambda}, x Y_{\tau}, v \mathbf{y}, u v-x y z Y_{1 \lambda_{\tau}}\right)$ and $\operatorname{rad}(\mathfrak{L})=$ $(x, y, z, u v)$. Thus grade $\left(I_{1}\left(d_{4}^{\prime}\right)\right)=\operatorname{grade}\left(I_{1}\left(d_{1}^{\prime}\right)\right) \geqslant 4$ and exactness is verified.

We are now in a position to prove that there are Gorenstein ideals of height four minimally generated by $n$ elements for any integer $n \geqslant 6$. By setting $u=v$ in the resolution $\mathbf{G}^{\prime}$ we see that such ideals can actually be found in $k[[x, y, z, v]]$. This result was conjectured by $J$. Watanabe [24] and has been independently verified by A. Iarrobino [private communication]. Kunz [16] has shown that $n=5$ is not possible.

COROLlary 2.5. There exist Gorenstein ideals of grade four minimally generated by $n$ elements for any integer $n \geqslant 6$. If $n$ is odd then such ideals can be found in the class $\operatorname{dim} \Lambda_{1}^{2}=3$.

Proof. We replace $R$ by its localization at the maximal ideal generated by the variables. For even $n$ the result is well known; one simply takes a hypersurface section of a codimension three Gorenstein ring $R / \mathfrak{B}$, where $\mathfrak{B}$ is minimally generated by $n-1$ elements. For odd $n$, we set $\tau=n-4$ and observe that $\mathbf{G}$ is a minimal resolution of $R / \mathfrak{B}$. It is clear by the structure of $\mathbf{G}$ that $\mathfrak{A}$ is a Gorenstein ideal of grade four. Now $\operatorname{dim} \Lambda_{1}^{2}$ is the maximum number of Koszul relations on the generators of $\mathfrak{A}$ that appear as columns of $d_{2}$ (see end of $\$ 1$ ). In $\mathbf{G}$ the first three columns of $d_{2}$ are Koszul relations on the first three generators $\mathbf{z}$ of $\mathfrak{A}$. By degree considerations all the other Koszul relations lie in $\mathrm{m}\left(\operatorname{im}\left(d_{2}\right)\right)$. Hence $\operatorname{dim} \Lambda_{1}^{2}=3$.

Since the generic ideals of Theorem 2.4 are perfect, they behave well under specialization.

COROLlary 2.6. If $R \rightarrow S$ is a homomorphism of Noetherian rings, and $\mathfrak{Y} S$ is a proper ideal of grade at least four, then $\mathfrak{X} S$ is a Gorenstein ideal of grade exactly four and $\mathbf{G} \otimes_{R} S$ is a free resolution of $S / \mathfrak{A} S$. If, further, $(S, \mathfrak{m})$ is local, and $\mathbf{G} \otimes S$ is a minimal resolution, then $\operatorname{dim} \Lambda_{1}^{2}=3$.

Proof. Except for the very last assertion this is an immediate consequence of Hochster [12, Proposition 6.14]. The last assertion follows from the explicit multiplication that we exhibit for $\mathbf{F}$ in Theorem 4.1. Assuming that $\mathfrak{A} S$ is proper and $\mathbf{G} \otimes S$ is a minimal resolution, then all the entires of $d_{2}$ are in $\mathrm{m}$. A quick glance at Theorem 4.1 shows that the set $\left\{e_{1} e_{2}, e_{1} e_{3}, e_{2} e_{3}\right\}$ is a basis for $\Lambda_{1}^{2}$; and all other products $e_{i} e_{j}$ are in $\mathfrak{m} F_{2}$. 
Theorem 2.4 yields sufficiently detailed structure for $\mathfrak{A}$ that we can prove a regularity result.

THEOREM 2.7. In the generic case $R / \mathfrak{A}$ is a normal domain.

Proof. Let $Q=\left(u, Y_{j}, \Sigma_{i=1}^{\tau} Y_{\alpha \beta i} t_{l i}\right)$ where $1 \leqslant j<\tau, 1<\alpha<\beta<\tau$, and $1 \leqslant l$ $<3$. Let $g$ denote any of the listed generators of $Q$. We will show that $(R / \mathfrak{Q})_{g}$ is regular for each $g$. If $P$ is a prime containing $\mathfrak{A}$, but ht $P<$ ht $Q$, then some $g$ is not in $P$ and $(R / \mathfrak{U})_{P}$ is regular. We will show that ht $Q \geqslant 6$; granted this, we see that $(R / \mathfrak{A})_{P}$ is regular if ht $P \leqslant 5$. Since $\mathfrak{A}$ is perfect, it is height-unmixed [14, Theorem 175], and ht $R_{R / \mathfrak{A}}(P / \mathfrak{U})=\mathrm{ht} P-\mathrm{ht} \mathfrak{A}$. Hence $R / \mathfrak{A}$ is regular in codimension one. As $R / \mathfrak{A}$ also satisfies the Serre condition $S_{2}$ it is a normal ring [20, Theorem 39] and hence a direct product of a finite number of normal domains [20, p. 116], [14, Theorems 167, 168]. If we assign the variables $u$ and $v$ the weights $\operatorname{deg} v=2$ and $\operatorname{deg} u=(\tau-1) / 2$, then $\mathfrak{A}$ is a homogeneous ideal. Therefore the spectrum of $R / \mathfrak{A}$ is connected and $R / \mathfrak{A}$ is a normal domain.

Claim. $(R / \mathfrak{A})_{g}$ is regular. There are essentially three cases. Whenever it is convenient we will denote the generators of $\mathfrak{A}$ by $a_{i}$, where $\left[a_{1}, a_{2}, a_{3}\right]=[u \mathbf{b}-\mathbf{y} A]$, $\left[a_{4}, \ldots, a_{3+\tau}\right]=[v \mathbf{y}-\mathbf{b} S]$, and $a_{4+\tau}=w-u v$. It is certainly clear that

$$
\left[a_{1}, \ldots, a_{\tau+4}\right] d_{2}=d_{1} d_{2}=0 \text {. }
$$

If $g=u$, then let $S$ be the regular ring $Z\left[x_{i j}(i \neq \tau+1), y_{\lambda \mu}, u, u^{-1}\right]$. We view $S$ as a subring of $R_{g}$ and define an $S$-algebra map $\phi: R_{g} \rightarrow S$ by $\phi(v)=u^{-1} w$ and $\phi\left(b_{j}\right)=u^{-1}(\mathbf{y} A)_{j}$ for $1 \leqslant j \leqslant 3$. Clearly $\phi$ is onto and $\operatorname{ker} \phi=\left(a_{1}, a_{2}, a_{3}, a_{\tau+4}\right) R_{g}$. We see from columns 4 through $\tau+3$ of $d_{2}$ and equation (8) that $\mathfrak{A}_{g}=\operatorname{ker} \phi$. Thus $(R / \mathfrak{A})_{g}$ is regular.

If $g=Y_{1}$, then we define a surjective $S$-algebra map

$$
\phi: R_{g} \rightarrow S=\mathbf{Z}\left[x_{i j}(i \neq 1), \quad y_{\lambda \mu}, u, Y_{1}^{-1}\right]
$$

by $\phi\left(x_{1 j}\right)=Y_{1}^{-1}\left(u b_{j}-\Sigma_{k=2}^{\tau} Y_{k} x_{k j}\right)$ for $j=1,2,3$ and $\phi(v)=Y_{1}^{-1}\left(\sum_{l=1}^{3} b_{l} s_{l 1}\right)$. Then ker $\phi=\left(a_{1}, a_{2}, a_{3}, a_{4}\right) R_{g}$. Using column 4 of $d_{2}$, we see that $a_{\tau+4}$ is in ker $\phi$. By Lemma 1.2

$$
d_{2}\left[\begin{array}{c}
0 \\
\vdots \\
0 \\
Y_{11 j} \\
Y_{21 j} \\
\vdots \\
Y_{\tau 1 j}
\end{array}\right]=\left[\begin{array}{c}
\sum t_{1 i} Y_{i 1 j} \\
\sum t_{2 i} Y_{i 1 j} \\
\sum t_{3 i} Y_{i 1 j} \\
Y_{j} \\
0 \\
\vdots \\
0 \\
-Y_{1} \\
0 \\
\vdots \\
0
\end{array}\right] \leftarrow \text { row } j+3
$$


and from this observation together with (8) we conclude that $a_{j+3}$ is in $\operatorname{ker} \phi$ for $2<j \leqslant \tau$. Thus $\mathfrak{A}_{g}=\operatorname{ker} \phi$ and $(R / \mathfrak{A})_{g}$ is regular. A similar argument shows $(R / \mathfrak{Q})_{g}$ regular for $g=Y_{2}, \ldots, Y_{\tau}$.

If $g=\sum_{i=1}^{\tau} Y_{\alpha \beta i} t_{l i}$ (with $\alpha, \beta, l$ fixed), then we show that $\mathfrak{A}_{g}=$ $\left(a_{m}, a_{n}, a_{\alpha+3}, a_{\beta+3}\right)_{g}$ where $m<n$ and $\{l, m, n\}=\{1,2,3\}$. Since $\Sigma_{i=1}^{\tau} y_{k i} Y_{\alpha \beta i}=$ $\delta_{k \alpha} Y_{\beta}-\delta_{k \beta} Y_{\alpha}$ (by Lemma 1.2) and

$$
d_{2}\left[\begin{array}{c}
0 \\
\vdots \\
0 \\
Y_{\alpha \beta 1} \\
\vdots \\
Y_{\alpha \beta r}
\end{array}\right]=\left[\begin{array}{c}
\Sigma t_{1 i} Y_{\alpha \beta i} \\
\Sigma t_{2 i} Y_{\alpha \beta i} \\
\Sigma t_{3 i} Y_{\alpha \beta i} \\
\Sigma y_{1 i} Y_{\alpha \beta i} \\
\vdots \\
\Sigma y_{r i} Y_{\alpha \beta i} \\
0
\end{array}\right]
$$

it follows that $a_{l}$ is in $\left(a_{m}, a_{n}, a_{\alpha+3}, a_{\beta+3}\right)_{g}$. The other computations are somewhat messier. For convenience let us take $l=1$. From

$$
d_{2}\left[\begin{array}{l}
0 \\
\vdots \\
0 \\
-Y_{\alpha \beta j} x_{\tau+13} \\
Y_{\alpha \beta j} x_{\tau+12} \\
\sum_{p=1}^{\tau} Y_{1 \alpha \beta j p} t_{1 p} \\
\vdots \\
\sum_{p=1}^{\tau} Y_{\tau \alpha \beta j p} t_{1 p}
\end{array}\right]=\left[\begin{array}{c}
0 \\
* \\
* \\
* \\
* \\
g \\
0
\end{array}\right] \begin{array}{r}
\leftarrow \operatorname{row} \alpha+3 \\
\leftarrow \operatorname{row} j+3 \\
\end{array}
$$

we conclude that $a_{j+3}$ is in $\left(a_{2}, a_{3}, a_{\alpha+3}, a_{\beta+3}\right)_{g}$ for $1<j<\tau$. Similarly, consideration of

$$
d_{2}\left[\begin{array}{l}
0 \\
\vdots \\
0 \\
-\sum_{i=1}^{\tau} Y_{\alpha \beta i} x_{i 3} \\
\sum_{i=1}^{\tau} Y_{\alpha \beta i} x_{i 2} \\
\sum_{p<q} Y_{1 \alpha \beta p q}\left(x_{p 2} x_{q 3}-x_{q 2} x_{p 3}\right) \\
\vdots \\
\sum_{p<q} \\
Y_{\tau \alpha \beta p q}\left(x_{p 2} x_{q 3}-x_{q 2} x_{p 3}\right)
\end{array}\right]
$$


yields that $a_{\tau+4}$ is in $\left(a_{2}, a_{3}, a_{\alpha+3}, a_{\beta+3}\right)_{g}$. Thus $\mathfrak{A}_{g}=\left(a_{m}, a_{n}, a_{\alpha+3}, a_{\beta+3}\right)_{g}$. We now rewrite the four generators as follows:

$$
\begin{aligned}
a_{3+\alpha} & =v Y_{\alpha}-\sum_{k=1}^{3} b_{k} s_{k \alpha}=-\sum_{1<i<j<\tau} Y_{\alpha i j} d_{i j \tau+1}+v Y_{\alpha} \\
& =-\sum_{i=1}^{\tau} Y_{\alpha \beta i} d_{\beta i \tau+1}+v Y_{\alpha}-\sum_{i, j \neq \beta} Y_{\alpha i j} d_{i j \tau+1} \\
& =\sum_{i=1}^{\tau} Y_{\alpha \beta i} \sum_{k=1}^{3}(-1)^{k} x_{\beta k} t_{k i}+\cdots \\
& =(-1)^{l} g x_{\beta l}+p_{\alpha}
\end{aligned}
$$

where $p_{\alpha}$ does not involve $x_{\alpha l}, x_{\beta l}, y_{\alpha \beta}, u ; a_{3+\beta}=(-1)^{l} g x_{\alpha l}+p_{\beta}$ where $p_{\beta}$ satisfies the same condition;

$$
\begin{gathered}
b_{m} a_{n}-b_{n} a_{m}=\sum_{k=1}^{\tau} Y_{k}\left|\begin{array}{cc}
x_{k m} & x_{k n} \\
b_{m} & b_{n}
\end{array}\right|=(-1)^{l+1} \sum_{k=1}^{\tau} Y_{k} t_{l k} \\
=(-1)^{l+1} \sum_{k=1}^{\tau} \sum_{i=1}^{\tau} y_{i \beta} Y_{i k \beta} t_{l k} \\
=(-1)^{l} y_{\alpha \beta} g+q, \\
\left(\sum_{i=1}^{\tau} Y_{\alpha \beta i} x_{i m}\right) a_{n}-\left(\sum_{i=1}^{\tau} Y_{\alpha \beta i} x_{i n}\right) a_{m}=u g+r
\end{gathered}
$$

where, again, $q$ and $r$ do not involve $x_{\alpha l}, x_{\beta l}, y_{\alpha \beta}, u$. Since

$$
g=\sum_{i=1}^{\tau} Y_{\alpha \beta i} t_{l i}=(-1)^{l}\left|\begin{array}{ll}
b_{m} & b_{n} \\
\sum Y_{\alpha \beta i} x_{i m} & \sum Y_{\alpha \beta i} x_{i n}
\end{array}\right|
$$

is invertible, we conclude that

$$
(R / \mathfrak{A})_{g} \simeq \mathbf{Z}\left[x_{i j}(i j \neq \alpha l \text { or } \beta l), y_{\lambda \mu}(\lambda \mu \neq \alpha \beta), v, g^{-1}\right],
$$

which is regular.

To conclude the proof we demonstrate that ht $Q \geqslant 6$. This can be accomplished by invoking the argument used in the proof of Theorem 2.3. Set all $x_{i j}=0$ except for $x_{22}=x_{2}, x_{33}=x_{3}, x_{\tau+11}=b_{1}$, and $x_{\tau+13}=b_{3}$. Pass to the factor ring $R^{\prime}=$ $R / \mathfrak{p}$, and let $Q^{\prime}=(Q+\mathfrak{p}) / \mathfrak{p}$. As before, $\mathrm{ht}_{R^{\prime}} Q^{\prime} \leqslant \mathrm{ht}_{R} Q$. In $Q^{\prime}$ some of the generators have a simpler form:

$$
\begin{aligned}
& \sum Y_{\alpha \beta i} t_{1 i}=-Y_{\alpha \beta 2} b_{3} x_{2}, \\
& \sum Y_{\alpha \beta i} t_{2 i}=-Y_{\alpha \beta 3} b_{1} x_{3}, \\
& \sum Y_{\alpha \beta i} t_{3 i}=Y_{\alpha \beta 2} b_{1} x_{2} .
\end{aligned}
$$

Let $P$ be a prime of $R^{\prime}$ containing $Q^{\prime}$. The ideal $\left(Y_{\alpha \beta k}\right)$ with $k$ fixed and $\alpha, \beta$ arbitrary is the ideal of $(\tau-3) \times(\tau-3)$ order pfaffians of a generic $(\tau-1) \times$ $(\tau-1)$ alternating matrix. It is well known that $\operatorname{ht}\left(Y_{\alpha \beta k}\right)=6$; see for instance Kleppe [15]. Thus if $\left(Y_{\alpha \beta k}\right)$ is contained in $P$ for some $k$, then ht $P \geqslant 6$. Otherwise $\left(u, \mathbf{y}, b_{3} x_{2}, b_{1} x_{3}, b_{1} x_{2}\right)$ is contained in $P$, and again ht $P>6$. 
Remark. Stronger results can be proved for small $\tau$. For $\tau=5$ or 7 the generic ring $R / \mathfrak{A}$ is regular in codimension two. In the case $\tau=3$ we can take $Q$ to be the ideal generated by $u, v, y$, and all $2 \times 2$ minors of the $4 \times 3$ matrix $X$; then one can show that $R / \mathfrak{A}$ is regular in codimension six.

3. Examples, a construction, and linkage. In this section we present several specializations that illustrate Corollary 2.6.

EXAMPLE 3.1. In the notation of the previous section, if one sets $X=0$ (so that $Z, S, T$, and $w$ vanish) and $v=1$, then $\mathfrak{A}=(\mathbf{y}, u)$ is the generic example of an even generated hypersurface section. In this case $\mathfrak{A}$ is minimally generated by $\tau+1$ elements and $\operatorname{dim} \Lambda_{1}^{2}=\tau=(1 / 2) \operatorname{dim} \Lambda_{2}$ if $\tau \geqslant 5$. (If $\tau=3$, then $\Lambda_{1}^{2}=\Lambda_{2}$ and $\mathfrak{A}$ is a complete intersection.)

Another specialization, and in fact the actual starting point in our discovery of the complex (6), arises from a construction of grade four Gorenstein ideals out of perfect almost complete intersections of grade three and their associated canonical modules; see Example 3.7. The fundamental tools involved come from the linkage theory of perfect ideals as presented in Buchsbaum and Eisenbud [4, $\$ \$ 5$ and 6] or Peskine and Szpiro [21, §§1 and 2].

We begin by showing how Gorenstein ideals of grade $g+1$ can be constructed from perfect ideals of grade $g$ and their canonical modules. Let $(R, \mathfrak{m})$ be a local Gorenstein ring. Suppose that $I$ is a perfect ideal of grade $g$ with depth $R / I>1$, type $I=\tau$, and $\mu(I)=\mu$. Let $K$ be the canonical module $\operatorname{Ext}^{8}(R / I, R)$. Assume that $I$ is a Gorenstein ideal locally at its minimal primes. Herzog and Kunz [10, Corollary 6.7] show that $K$ is isomorphic to an ideal $L / I$ of $R / I$ and that $L$ contains an element of $R$ that is regular on $R / I$.

LEMMA 3.2. With $I$ and $L$ as above, let $v$ be an element of $m$ that is regular on $R / I$. Let $\mathfrak{A}$ be the ideal $I+v L$. Then $\mathfrak{A}$ is a Gorenstein ideal of grade $g+1$ that is minimally generated by $\mu+\tau$ elements.

Proof. Let $\mathbf{P}$ be a minimal resolution of $R / I$. Since $I$ is perfect $\mathbf{P}^{*}$ is a minimal resolution of $K$. There is a comparison map $\psi: \mathbf{P}^{*} \rightarrow \mathbf{P}$ which extends the injection $K \rightarrow \simeq L / I \rightarrow R / I$. The mapping cone $\mathrm{C}$ of $\vartheta \psi$ is acyclic and resolves $R / \mathfrak{A}$; hence $\operatorname{pd}_{R} R / \mathfrak{A} \leqslant g+1$. On the other hand, by hypothesis $\mathfrak{A}$ contains an element of $R$ which is regular on $R / I$. Thus

$$
g+1 \leqslant \operatorname{grade} \mathfrak{U} \leqslant \operatorname{pd}_{R} R / \mathfrak{A} \leqslant g+1,
$$

and $R / \mathfrak{A}$ is Cohen-Macaulay. The last nonzero module in the resolution $\mathbf{C}$ is $P_{0}^{*} \simeq R$. Therefore $R / \mathfrak{A}$ has type 1 and is Gorenstein. We choose $v$ to be in $\mathrm{m}$ and $\mathbf{C}$ to be the mapping cone of $v \psi$ in order to insure that $\mathbf{C}$ is a minimal resolution of $R / \mathfrak{A}$. In particular, $\mathfrak{A}$ is generated by $\mu+\tau$ elements.

REMARK 3.2a. If $L \neq R$ we need not take $v$ to be in $\mathrm{m}$. Of course if $v$ is not in $\mathrm{m}$, the resolution $\mathbf{C}$ may not be minimal.

In order to have a concrete realization of the ideal $L$ in Lemma 3.2 we need the theory of linkage. All of this material can be found in the literature; we have collected it together for convenience. Throughout this discussion $I$ and $J$ are 
unmixed (see Matsumura [20, Definition 16c]) ideals of grade $g$ in a CohenMacaulay local ring $(R, \mathfrak{m})$, and $\mathrm{z}$ is a regular sequence of length $g$ contained in $I \cap J$.

DEFINITION 3.3. (a) $I$ and $J$ are geometrically linked by $\mathrm{z}$ if $I$ and $J$ have no common components and $I \cap J=(\mathbf{z})$.

(b) $I$ and $J$ are algebraically linked by $\mathbf{z}$ if (z): $I=J$ and (z): $J=I$.

RemarK. If $I$ or $J$ is a perfect ideal and $R$ is Gorenstein, then (z): $I=J$ if and only if $(\mathrm{z}): J=I$. In this case both ideals are perfect. See [4, Proposition 5.1].

Proposition 3.4. (a) Geometric linkage implies algebraic linkage.

(b) If $(\mathbf{z}): I=J$, then the following statements are equivalent.

(i) $I$ and $J$ are geometrically linked by $\mathbf{z}$.

(ii) $I$ and $J$ have no common components.

(iii) $I_{P}=(\mathbf{z})_{P}$ for all associated primes $P$ of $R / I$ (and similarly for $J$ ).

(iv) $I \cap J=(\mathbf{z})$.

Proof. Part (a) is straightforward. For (b) it suffices to show that the last three statements are equivalent.

(ii) $\Leftrightarrow$ (iii). This equivalence follows readily from localization of (z): $I=J$ at the associated primes of $I$.

(iii) $\Rightarrow$ (iv). Since $I$ and $J$ have no common components $(I \cap J)_{P}=I_{P}=(\mathbf{z})_{P}$ for all $P$ in $\operatorname{Ass}(R / I)$ and $(I \cap J)_{P}=J_{P}=(\mathbf{z})_{P}$ for all $P$ in $\operatorname{Ass}(R / J)$. If $Q$ is minimal in $\operatorname{Supp}(I \cap J) /(\mathbf{z})$, then $\operatorname{depth}(R /(\mathbf{z}))_{Q}=0$. Thus $Q$ is a minimal prime of (z) and hence an associated prime of $I$ or $J$, which contradicts $(I \cap J)_{Q} \neq(\mathbf{z})_{Q}$.

(iv) $\Rightarrow$ (ii). If $I \cap J=(\mathbf{z})$ and (z): $I=J$, then $J=(I \cap J): I=J: I$. Hence $I$ is not contained in any associated prime of $J$.

Let $I$ and $J$ be geometrically linked ideals of grade $g$ in a Gorenstein local ring $(R, \mathrm{~m})$. Peskine and Szpiro [21, Remarque 1.4] note that $I+J$ is a Gorenstein ideal of grade $g+1$. In Corollary 3.5 we offer another argument: we produce a projective resolution for $R /(I+w J)$ provided one is known for $R / I$.

COROLLARY 3.5. Let $(R, \mathfrak{m})$ be a Gorenstein local ring and I and $J$ be perfect ideals of grade $g$. Assume that $I$ and $J$ are geometrically linked and that $v$ is a regular element on $R / I$. Then $I+v J$ is a Gorenstein ideal of grade $g+1$.

Proof. Let $\mathrm{z}$ be the regular sequence $I \cap J$. It is well known [2, Proposition 2.9] that the canonical module $K=\operatorname{Ext}_{R}^{8}(R / I, R)$ is isomorphic to $((\mathbf{z}): I) /(\mathbf{z})=J /(\mathbf{z})$. Since $I \cap J=(\mathbf{z})$ there is an embedding $K \rightarrow(J+I) / I \subset R / I$, and we can take $L=I+J$ in Lemma 3.2 or $3.2 \mathrm{a}$.

We now apply Corollary 3.5 in the situation that $I$ is a perfect almost complete intersection of grade three. Since the structure of the resolution is known [4], we are able to draw strong conclusions about the Gorenstein ideal $\mathfrak{A}=I+\mathfrak{v}$ and the structure of its resolution. We begin by showing how almost complete intersections of odd type arise. 
Proposition 3.6 [4, Proposition 5.3]. Let $(R, \mathfrak{m})$ be a Gorenstein local ring and I and $J$ be perfect ideals of grade three which are algebraically linked by a regular sequence $\mathrm{z}=z_{1}, z_{2}, z_{3}$. Let $\tau$ be the type of I. If $J$ is a Gorenstein ideal and $\mathrm{z} \subset \mathrm{mJ}$, then $I$ is an almost complete intersection, $\mathrm{z}$ forms part of a minimal set of generators for $I$, and $\tau$ is the odd number $\mu(J)$.

Proof. For future use we record the proof in the notation of $\$ 2$. The type of $I$ is equal to the minimal number of generators of the canonical module

$$
K=\operatorname{Ext}^{3}(R / I, R) \simeq(\mathbf{z}: I) / \mathbf{z}=J / \mathbf{z} .
$$

Thus $\tau=\mu(J)$, which is odd, and there is a $\tau \times \tau$ alternating matrix $Y$ such that $(\mathbf{y})=J$. Since $\mathrm{z} \subset \mathrm{m} J$, there is a $\tau \times 3$ matrix $A$ with entries in $\mathfrak{m}$ so that $\mathrm{z}=-\mathbf{y} A$. We define $w, S$, and $Z$ as in Theorem 2.1, and let $\mathbf{b}=0$. (So $B$ and $T$ also vanish.)

The natural map $R /(\mathbf{z}) \rightarrow R / J$ lifts to the following comparison of resolutions

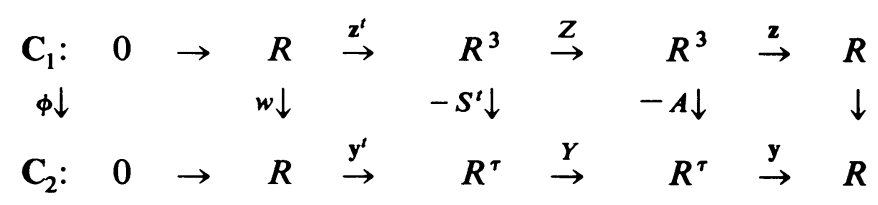

in which commutativity is a consequence of Lemma 2.2. (Commutativity can also be verified by observing that $\phi$ is an algebra map.) The mapping cone of $\phi^{*}$ : $\mathrm{C}_{2}^{*} \rightarrow \mathrm{C}_{1}^{*}$ is a resolution of $R /((\mathbf{z}): J)=R / I$, and, after removal of the extraneous summand at the end, is simply

$$
\text { P: } 0 \rightarrow R^{\tau} \stackrel{d_{3}}{\rightarrow} R^{3+\tau} \stackrel{d_{2}}{\rightarrow} R^{4} \stackrel{d_{1}}{\rightarrow} R
$$

where $d_{1}=[\mathbf{z}, w], d_{3}=[A, Y]^{t}$, and

$$
d_{2}=\left[\begin{array}{ll}
Z & S \\
0 & \mathbf{y}
\end{array}\right]
$$

Since all the matrices involved have all entries in $\mathrm{m}$, the resolution $\mathbf{P}$ is minimal.

RemarK. Buchsbaum and Eisenbud also show that if $I$ is an almost complete intersection of odd type $\tau$, then there exists a regular sequence $\mathbf{z}=z_{1}, z_{2}, z_{3}$ such that $\mathrm{z}$ forms part of a minimal generating set for $I$ and $\mathrm{z} \subset \mathfrak{m} J$ where $J$ is the Gorenstein ideal (z): $I$.

In Example 3.7 we combine the hypotheses of Corollary 3.5 and Proposition 3.6. We require that $J$ be a perfect Gorenstein ideal of grade three which is geometrically linked to $I$ by $\mathbf{z}$. We also insist that $\mathbf{z}$ be contained in $\mathrm{mJ}$. There are at least two ways to meet these conditions:

(1) If $\mathrm{z} \subset \mathrm{m} J$ and $I=(\mathrm{z}): J$, then by Proposition 3.6 we know $\mathrm{z}$ is not in $\mathrm{m} I$ and hence $J$ is not contained in $I$. If $I$ is prime (as it is in the generic case [4, Proposition 6.3]), then $I$ and $J$ have no common components and are thus geometrically linked.

(2) Suppose $R$ contains an infinite field and $J$ is a perfect ideal of grade $g$ which is not m-primary. If $R / J$ is a generic complete intersection then a general position argument (see [21, Lemme 3.8]) produces a length $g$ regular sequence $\mathrm{z}$ with $\mathrm{z} \subset \mathrm{mJ}$ 
and $(\mathbf{z})_{P}=J_{P}$ for all associated primes $P$ of $R / J$. Then $J$ and $I=(\mathbf{z}): J$ are geometrically linked.

We now come to the main result of this section.

EXAMPLE 3.7. Let $I$ and $J$ be geometrically linked perfect ideals of grade three in a Gorenstein local ring $(R, \mathrm{~m})$. Suppose that $J$ is a Gorenstein ideal and $I \cap J \subset$ $\mathfrak{m} J$. Let $v$ be an element of $m$ that is regular on $R / I$. Then $\mathfrak{A}=I+v J$ is a Gorenstein ideal of grade four with $\operatorname{dim} \Lambda_{1}^{2}=3$. Moreover, $R / \mathfrak{A}$ has a minimal resolution of form (6).

Proof. Let $\mathbf{z}$ generate the complete intersection $I \cap J$, and let $\mathbf{P}$ be the minimal resolution of $R / I$ given in (9). We record an explicit comparison map $\psi: \mathbf{P}^{*} \rightarrow \mathbf{P}$

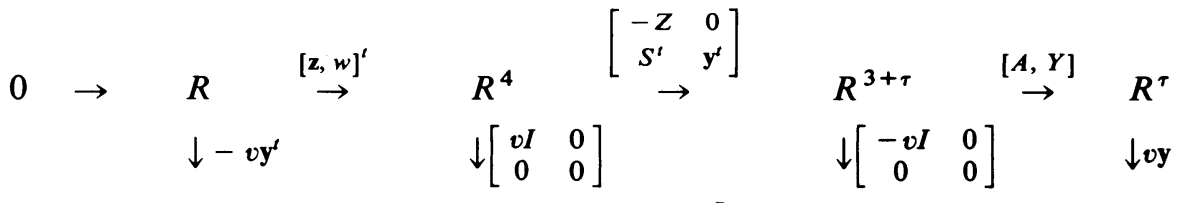

$$
\begin{aligned}
& 0 \rightarrow R^{\tau} \stackrel{[A, Y]^{t}}{\rightarrow} \quad R^{3+\tau} \quad \stackrel{\left[\begin{array}{ll}
Z & S \\
0 & \mathbf{y}
\end{array}\right]}{\rightarrow} \quad R^{4} \stackrel{[\mathbf{z}, w]}{\rightarrow} \quad R
\end{aligned}
$$

that lifts the embedding $v(I+J) / I \subset R / I$. The mapping cone of $\psi$ is the complex

$$
\mathrm{C}: 0 \rightarrow R \stackrel{d_{4}}{\rightarrow} R^{r+\tau} \stackrel{d_{3}}{\rightarrow} R^{2(3+\tau)} \stackrel{d_{2}}{\rightarrow} R^{4+\tau} \stackrel{d_{1}}{\rightarrow} R
$$

with

$$
\begin{array}{ll}
d_{1}=[\mathbf{z}, v \mathbf{y}, w], & d_{2}=\left[\begin{array}{llll}
Z & S & v I & 0 \\
0 & 0 & A & Y \\
0 & \mathbf{y} & 0 & 0
\end{array}\right], \\
d_{3}=\left[\begin{array}{ll}
0 & I \\
I & 0
\end{array}\right] d_{2}^{t}, & d_{4}=d_{1}^{t} .
\end{array}
$$

Now $\mathfrak{A}=I+v L=I+v J$ and $\mathbf{C}$ is exact by Corollary 3.5 . In fact we recognize $\mathrm{C}$ as a specialization of the generic resolution $\mathbf{G}$, and by Corollary 2.6 we conclude that $\operatorname{dim} \Lambda_{1}^{2}=3$.

REMARK. A grade four Gorenstein ideal which is obtained from the construction of Lemma 3.2 need not have $\operatorname{dim} \Lambda_{1}^{2}=3$. The almost complete intersections of type two yield hypersurface sections in the class $\operatorname{dim} \Lambda_{1}^{2}=(1 / 2) \operatorname{dim} \Lambda_{2}$ and there are examples in which $\mathfrak{A}$ falls in the class $\Lambda_{1}^{2}=0$.

In the beginning of $\$ 2$ we mentioned that some rings of form $k\left[T^{n_{1}}, T^{n_{2}}, \ldots, T^{n_{5}}\right]$, where $\left\langle n_{1}, \ldots, n_{5}\right\rangle$ is a symmetric semigroup, give rise to grade four Gorenstein ideals in the class $\operatorname{dim} \Lambda_{1}^{2}=3$. Here is one example.

EXAMPLE 3.8. Let $\mathfrak{A}$ be the kernel of the map

$$
R=k[A, b, C, D, E] \rightarrow k\left[T^{9}, T^{10}, T^{11}, T^{13}, T^{17}\right]
$$


with $A \mapsto T^{9}$, etc. Then $\mathfrak{A}$ is generated by $B^{2}-A C, C^{2}-A D, D^{2}-A E, A^{3}-$ $B E, A^{2} B-C E, E^{2}-B C D$, and $D E-A B C$. If we take $\mathbf{y}=[-B,-C, E]$, $u=-D, v=E$, and

$$
X=\left[\begin{array}{rrr}
B & 0 & 0 \\
-A & C & 0 \\
0 & 0 & A \\
0 & A & -D
\end{array}\right],
$$

then this specialization of $\mathbf{G}$ is a resolution of $R / \mathfrak{A}$.

Finally we offer one more piece of evidence that any grade four Gorenstein ideal in the class $\operatorname{dim} \Lambda_{1}^{2}=3$ has a resolution that can be obtained by a specialization of G.

EXAMPLE 3.9. Let $R=k[[A, \ldots, G]]$ and let $I$ be the height 3, type 2, 5-generated perfect ideal generated by $A^{2}-B D, B^{2}-C E, C^{2}-A B F, A C-D E F$, and $B C-A E F$. This ideal is example (f) in Herzog and Kunz [11]. Since $I$ is not an almost complete intersection (indeed it has deviation 2), we cannot use it in the context of Example 3.7. However, the canonical module of $R / I$ is isomorphic to $[(C, F)+I] / I$; hence by Lemma 3.2, $\mathfrak{A}=I+(C, F) G$ is a grade four Gorenstein ideal. It turns out that $\mathfrak{A}$ is in the class $\operatorname{dim} \Lambda_{1}^{2}=3$. If we let $\mathbf{y}=[-A,-C,-G]$, $u=B, v=-C$, and

$$
X=\left[\begin{array}{rrr}
A & 0 & 0 \\
0 & -E & 0 \\
0 & 0 & -F \\
-D & B & 0
\end{array}\right]
$$

then this specialization of $\mathbf{G}$ is a resolution of $R / \mathfrak{A}$.

4. Algebra structure on F. In [17] we proved that if $(R, \mathfrak{m})$ is a Gorenstein local ring in which 2 is a unit, and if $\mathfrak{A}$ is a Gorenstein ideal of codimension four, then every minimal free resolution of $R / \mathfrak{A}$ admits the structure of an associative, differential, graded commutative algebra (DGC algebra). We did not, however, exhibit explicit multiplication tables. In this section we give an explicit DGC structure for complexes $\mathbf{F}$ of form (6), without restriction on the commutative ring $R$. It will be apparent from this result that $\operatorname{dim} \Lambda_{1}^{2}=3$ if $R$ is local, which will confirm the assertion made in Corollary 2.6. We refer the reader to [17] for a full discussion of the characterization and existence of DGC structures on $\mathbf{F}$.

Given a complex $\mathbf{F}$ of form (6), let us denote the basis elements of $F_{1}$ by $e_{1}, \ldots, e_{\tau+4}$, of $F_{2}$ by $f_{1}, \ldots, f_{\tau+3}, f_{1}^{\prime}, \ldots, f_{\tau+3}^{\prime}$, and of $F_{3}$ by $g_{1}, \ldots, g_{\tau+4}$. There is an isomorphism of complexes $\mathbf{F} \rightarrow \mathbf{F}^{*}$

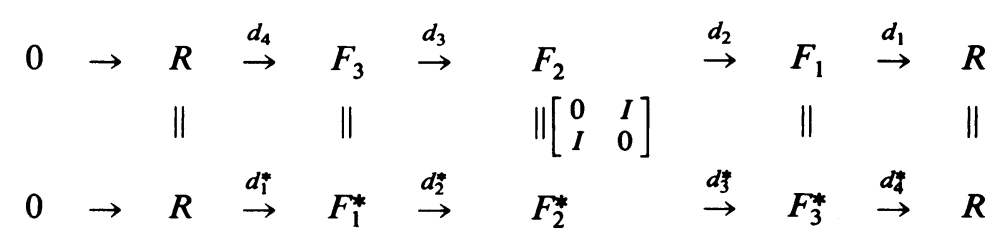


and the bases we have chosen are dual under the induced perfect pairings $F_{1} \times F_{3} \rightarrow R$ and $F_{2} \times F_{2} \rightarrow R$. A DGC structure on $\mathbf{F}$ can be constructed by first defining the products $e_{i} g_{j}=\delta_{i j}, f_{i} f_{j}=f_{i}^{\prime} f_{j}^{\prime}=0$, and $f_{i} f_{j}^{\prime}=-\delta_{i j}$. Then we only need to define the products of elements of degree one to determine completely the DGC structure. In particular, if $x_{i}$ is in $F_{i}$ for $i=1,2$, then the element $x_{1} x_{2}$ in $F_{3}$ is determined once the multiplication $F_{1} \otimes F_{1} \rightarrow F_{2}$ is known because $e_{j}\left(x_{1} x_{2}\right)=$ $\left(e_{j} x_{1}\right) x_{2}$ for $j=1, \ldots, \tau+4$.

THEOREM 4.1. The products $e_{i} e_{j}$ defined below endow a complex $\mathbf{F}$ of form (6) with a DGC algebra structure.

$$
\begin{gathered}
e_{1} e_{2}=f_{3}, \quad e_{1} e_{3}=-f_{2}, \quad e_{2} e_{3}=f_{1}, \\
e_{i} e_{j+3}=x_{\tau+1, i} f_{j+3}-Y_{j} f_{i}^{\prime}+\sum_{k=1}^{\tau}\left(\sum_{l=1}^{\tau} x_{l i} Y_{k l j}\right) f_{k+3}^{\prime} \\
\text { for } 1<i<3 \text { and } 1 \leqslant j \leqslant \tau, \\
e_{i} e_{\tau+4}=-\sum_{k=1}^{\tau} x_{k i} f_{k+3}+u f_{i}^{\prime} \quad \text { for } 1 \leqslant i \leqslant 3, \\
e_{i+3} e_{j+3}=\sum_{k=1}^{3}\left(\sum_{l=1}^{\tau} t_{k l} Y_{l i j}\right) f_{k}^{\prime}-\sum_{k=1}^{\tau}\left(v Y_{k i j}+\sum_{\alpha<\beta} d_{\alpha \beta r+1} Y_{i k j \beta \beta}\right) f_{k+3}^{\prime} \\
e_{i+3} e_{\tau+4}=v f_{i+3}-\sum_{k=1}^{3} s_{k i} f_{k}^{\prime}-\sum_{k=1}^{\tau} n_{k i} f_{k+3}^{\prime} \quad \text { for } 1 \leqslant i<j \leqslant \tau,
\end{gathered}
$$

and

$$
\begin{cases}e_{i} e_{j}=-e_{j} e_{i} & \text { for } i>j \\ e_{i} e_{j}=0 & \text { for } i=j\end{cases}
$$

(The matrices $T$ and $S$ are defined before Theorem 2.1; the matrix $N$ is defined in the proof of Lemma 2.2 just before line (7).)

Proof. The products $e_{i} e_{j}$ must be defined to be compatible with the differential on $\mathbf{F}$, namely

$$
d_{2}\left(e_{i} e_{j}\right)=d_{1}\left(e_{i}\right) e_{j}-e_{i} d_{1}\left(e_{j}\right) .
$$

Since $d_{1}$ annihilates the right-hand side it is clear that one can choose some element in $F_{2}$ to serve as $e_{i} e_{j}$. The problem is to do this in such a way as to guarantee that the differential is compatible with the multiplication in higher degrees, and so that associativity holds in general. In [17, Proposition 3.1] we show that if

$$
\left(d x_{1}\right)\left(x_{2} y_{2}\right)=\left(x_{1} d x_{2}\right) y_{2}+\left(x_{1} d y_{2}\right) x_{2}
$$

for all $x_{1}$ in $F_{1}$ and $x_{2}, y_{2}$ in $F_{2}$ and (10) holds, then the multiplication defines a DGC structure on F. Of course one only needs to check (7) for the basis elements of $F_{1}$ and $F_{2}$. While it is feasible, if tedious, to check (10) and (11) case by case, we 
actually express the multiplication in table form (as matrices of "structure constants"), partition these in a systematic way, and verify matrix identities equivalent to (10) and (11). As this analysis, too, is rather tedious we omit it.

One should note that we have not cited the existence results (Theorems 2.4 and 4.3) of [17] in which invertibility of 2 was assumed. The form of $F$ allows us to take the conclusion of Theorem 2.4 for granted, and only the sufficiency criterion of Proposition 3.1 needs to be invoked. An inspection of the proof of Proposition 3.1 reveals that $\mathbf{F}$ need only be a complex and that $R$ can be any commutative ring. All we require is that $\mathfrak{A}$ be generated by elements that are not zero-divisors in $R$ and that $0 \rightarrow R \rightarrow F_{1}^{*} \rightarrow F_{2}^{*}$ be exact, conditions that clearly hold in the generic case. But if the generic complex $\mathbf{G}$ admits a DGC structure, so does any specialization.

As we noted in the discussion just prior to the theorem, the multiplication $F_{1} \otimes F_{2} \rightarrow F_{3}$ has been forced, and thus we can compute the additional invariant $\operatorname{dim} \Lambda_{1} \Lambda_{2}$ in the local case. First let us consider the situation in which $F$ is a minimal resolution of $R / \mathfrak{A}$ over the local ring $(R, \mathfrak{m})$. We have the multiplication tables

\begin{tabular}{l|rrr} 
& $e_{1}$ & $e_{2}$ & $e_{3}$ \\
\hline$e_{1}$ & 0 & $f_{3}$ & $-f_{2}$ \\
$e_{2}$ & $-f_{3}$ & 0 & $f_{1}$ \\
$e_{3}$ & $f_{2}$ & $-f_{1}$ & 0
\end{tabular}

\begin{tabular}{l|rrr} 
& $f_{1}^{\prime}$ & $f_{2}^{\prime}$ & $f_{3}^{\prime}$ \\
\hline$e_{1}$ & 0 & $-g_{3}$ & $g_{2}$ \\
$e_{2}$ & $g_{3}$ & 0 & $-g_{1}$ \\
$e_{3}$ & $-g_{2}$ & $g_{1}$ & 0
\end{tabular}

All other products in $F_{1}^{2}$ and $F_{1} F_{2}$ lie in $\mathrm{m}_{2}$ and $\mathfrak{m} F_{3}$, respectively. Hence $\operatorname{dim} \Lambda_{1} \Lambda_{2}=\operatorname{dim} \Lambda_{1}^{2}=3$.

Now let us suppose that $\mathbf{F}$ is a resolution of $R / \mathfrak{A}$, but not minimal. For instance in Example 3.1 we have $\operatorname{dim} \Lambda_{1}^{2}=(1 / 2) \operatorname{dim} \Lambda_{2}$ and $\Lambda_{1} \Lambda_{2}=\Lambda_{3}$. The following example is more subtle.

EXAMPLE 4.2 (NOTATION AS IN COROLlaRY 2.6). Specialize the generic resolution $\mathbf{G}$ over the polynomial ring $R$ by setting $x_{11}=1$. Let $(S, \mathfrak{m}, k)$ be the localization of $R /\left(x_{11}-1\right)$ at the maximal ideal $m$ generated by the remaining variables, and let $\mathbf{F}=\mathbf{G} \otimes S$. Then $\mathbf{F}$ is a nonminimal free resolution of $S / \mathfrak{A} S$. By Theorem 4.1 the set $\left\{e_{1} e_{2}, e_{1} e_{3}, e_{1} e_{\tau+4}\right\}$, or essentially $\left\{f_{3}, f_{2}, f_{4}\right\}$ is a basis for $F_{1}^{2} \otimes k$ and $\left\{g_{1}, g_{2}, g_{3}, g_{\tau+4}\right\}$ is a basis for $F_{1} F_{2} \otimes k$. Now $\Lambda_{.}=H .(F \otimes k)$ and since $x_{11}=1$ in $S$, we find that $\Lambda_{1}=\left(F_{1} / S e_{4}\right) \otimes k, \Lambda_{2}=\left(F_{2} / S f_{1} \oplus S f_{1}^{\prime}\right) \otimes k$, and $\Lambda_{3}=\left(F_{3} / S g_{4}\right)$ $\otimes k$. Then $\operatorname{dim} \Lambda_{1}^{2}=3$ and $\operatorname{dim} \Lambda_{1} \Lambda_{2}=4$.

REMARK. If $I$ is a perfect almost complete intersection of grade three and even type $\tau \geqslant 4$, then the construction that led to Example 3.7 will lead in this case to an even-generated Gorenstein ideal $\mathfrak{A}$ of grade four with $\operatorname{dim} \Lambda_{1}^{2}=3$ and $\operatorname{dim} \Lambda_{1} \Lambda_{2}=$ 4. (The resolution of $R / \mathfrak{A}$ has essentially the same form as $F$ in the example above.) Hence for each even $n \geqslant 8$ there are $n$-generated codimension four Gorenstein ideals that are not hypersurface sections of codimension three Gorenstein ideals.

As a further application of Theorem 4.1 it is now quite a simple matter to compute products that involve at least two of $e_{1}, e_{2}, e_{3}$. For instance $e_{1} e_{2} e_{3} e_{j+3}=Y_{j}$ 
since only the term with $f_{3} f_{3}^{\prime}$ survives. In this manner one can show that a portion of the matrix $d_{2}$ can be recovered from the algebra structure, namely

$$
\left[\begin{array}{c}
S \\
u I \\
\mathbf{y}
\end{array}\right]=\left[\begin{array}{rccc}
e_{2} e_{3} e_{4} e_{\tau+4} & e_{2} e_{3} e_{5} e_{\tau+4} & \cdots & e_{2} e_{3} e_{\tau+3} e_{\tau+4} \\
-e_{1} e_{3} e_{4} e_{\tau+4} & -e_{1} e_{3} e_{5} e_{\tau+4} & \cdots & -e_{1} e_{3} e_{\tau+3} e_{\tau+4} \\
e_{1} e_{2} e_{4} e_{\tau+4} & e_{1} e_{2} e_{5} e_{\tau+4} & \cdots & e_{1} e_{2} e_{\tau+3} e_{\tau+4} \\
-e_{1} e_{2} e_{3} e_{\tau+4} & 0 & \cdots & 0 \\
\vdots & \vdots & & \vdots \\
0 & -e_{1} e_{2} e_{3} e_{\tau+4} & \cdots & \vdots \\
\vdots & \vdots & & 0 \\
0 & 0 & \cdots & -e_{1} e_{2} e_{3} e_{\tau+4} \\
e_{1} e_{2} e_{3} e_{4} & e_{1} e_{2} e_{3} e_{5} & \cdots & e_{1} e_{2} e_{3} e_{\tau+3}
\end{array}\right]
$$

The columns clearly represent relations on the generators $d e_{1}, \ldots, d e_{\tau+4}$ of $\mathfrak{A}$; for example the first column represents the relation

$$
\begin{aligned}
0 & =d\left(e_{1} e_{2} e_{3} e_{4} e_{\tau+4}\right) \\
& =\sum_{i=1}^{4}(-1)^{i+1}\left(d e_{i}\right) e_{1} \cdots \hat{e}_{i} \cdots e_{4} e_{\tau+4}+\left(d e_{\tau+4}\right) e_{1} e_{2} e_{3} e_{4} .
\end{aligned}
$$

This suggests that if a DGC structure is available, then one will be able to prove a converse to Corollary 2.6 along the following lines: if $\mathfrak{A}$ is a Gorenstein ideal of grade four whose minimal resolution admits a DGC structure with $\operatorname{dim} \Lambda_{1}^{2}=3$, then $R / \mathfrak{U}$ has a minimal resolution of form (6), a specialization of the generic resolution $\mathbf{G}$. The difficulty appears to be in the selection of basis elements for the algebra $F$. If there is a basis whose multiplication table is given by (12), then we can prove the following result.

THEOREM. Let $\mathfrak{A}$ be a 7-generated Gorenstein ideal of grade four in a regular local ring $(R, \mathfrak{m})$, and let $\mathbf{F}$ be a DGC algebra which is a minimal free resolution of $R / \mathfrak{A}$. Assume that $\operatorname{dim} \Lambda_{1}^{2}=\operatorname{dim} \Lambda_{1} \Lambda_{2}=3$. If $e_{1}, e_{2}, e_{3}$ is the start of a basis for $F_{1}$ and $f_{1}$, $f_{2}, f_{3}, f_{1}^{\prime}, f_{2}^{\prime}, f_{3}^{\prime}$ is the start of a basis for $F_{2}$ with

(a) $e_{1} e_{2}=f_{3}, e_{2} e_{3}=f_{1}, e_{3} e_{1}=f_{2}$,

(b) $d e_{1}, d e_{2}, d e_{3}$ a regular sequence,

(c) $f_{i}^{\prime} f_{j}^{\prime}=0, f_{i} f_{j}^{\prime}=-\delta_{i j}$,

(d) $e_{i} f_{j}^{\prime}+e_{j} f_{i}^{\prime}=0, e_{i} f_{i}^{\prime}=0$ for $1 \leqslant i, j \leqslant 3$,

then $\mathbf{F}$ is isomorphic to a resolution of form (6).

Since the result is incomplete and the proof is rather long, we omit it. However, we observe that most of the statement is merely a recording of the notation that is required to state the only real restriction, which is condition (d). The hypothesis $\operatorname{dim} \Lambda_{1}^{2}=3$ implies that there are elements $e_{1}, e_{2}, e_{3}$, and $f_{1}, f_{2}, f_{3}$ that satisfy condition (a). Condition (b) is met by the usual prime avoidance techniques. If 2 is a unit of $R$, then $\left(f_{1}, f_{2}, f_{3}\right)$ has a hyperbolic complement. In other words, there are elements $f_{1}^{\prime}, f_{2}^{\prime}, f_{3}^{\prime}$ that satisfy condition (c). The equations of condition (d) are satisfied in $\mathbf{F} / \mathbf{m F}$. The theorem asserts that whenever these equations can be successfully lifted to $F$, then $F$ is isomorphic to a resolution of form (6). 


\section{REFERENCES}

1. E. Artin, Geometric algebra, Interscience Tracts in Pure and Appl. Math., vol. 3, Interscience, London, 1957.

2. H. Bass, On the ubiquity of Gorenstein rings, Math. Z. 82 (1963), 8-28.

3. H. Bresinsky, Monomial Gorenstein ideals, Manuscripta Math. 29 (1979), 159-181.

4. D. Buchsbaum and D. Eisenbud, Algebra structures for finite free resolutions, and some structure theorems for ideals of codimension 3, Amer. J. Math. 99 (1977), 447-485.

5. What makes a complex exact?, J. Algebra 25 (1973), 259-268.

6. H. Cartan and S. Eilenberg, Homological algebra, Princeton Univ. Press, Princeton, N. J., 1956.

7. T. Gulliksen and $\mathrm{O}$. Negard, Un complexe résolvant pour certains ideaux determinantiels, C.R. Acad. Sci. Paris Sér. A 274 (1972), 16-18.

8. J. Herzog, Certain complexes associated to a sequence and a matrix, Manuscripta Math. 12 (1974), 217-248.

9. , Komplexe, Auflösungen und Dualität in der lokalen Algebra, Habilitationsschrift Regensburg, 1973.

10. J. Herzog and E. Kunz, Der kanonische Modul eines Cohen-Macaulay Rings, Lecture Notes in Math., vol. 238, Springer-Verlag, Berlin and New York, 1971.

11. On the deviation and the type of a Cohen-Macaulay ring, Manuscripta Math. 9 (1973), 383-388.

12. M. Hochster, Topics in the homological theory of modules over commutative rings, Regional Conf. Ser. Math. vol. 24, Amer. Math. Soc., Providence, R. I., 1975.

13. M. Hochster and J. Roberts, Rings of invariants of reductive groups acting on regular rings are Cohen-Macaulay, Adv. in Math. 13 (1974), 115-175.

14. I. Kaplansky, Commutative rings, rev. ed., Univ. Chicago Press, Chicago, Ill., 1974.

15. H. Kleppe, Deformation of pfaffian schemes, J. Algebra 53 (1978), 84-92.

16. E. Kunz, Almost complete intersections are not Gorenstein rings, J. Algebra 28 (1974), 111-115.

17. A. Kustin and M. Miller, Algebra structures on minimal resolutions of Gorenstein rings of embedding codimension four, Math. Z. 173 (1980), 171-184.

18. Algebra structures on minimal resolutions of Gorenstein rings, Regional Conference at George Mason University (August, 1979) (to appear).

19. S. Lang, Algebra, Addison-Wesley, New York, 1971.

20. H. Matsumura, Commutative algebra, Benjamin, New York, 1970.

21. C. Peskine and L. Szpiro, Liaison des variétés algébriques. I, Invent. Math. 26 (1974), 271-302.

22. J.-P. Serre, Algèbre locale-multiplicités, Lecture Notes in Math., vol. 11, 2nd ed., Springer-Verlag, Berlin and New York, 1965.

23. R. Stanley, Invariants of finite groups and their applications to combinatorics, Bull. Amer. Math. Soc. (N. S.) 1 (1979), 475-511.

24. J. Watanabe, $A$ note on Gorenstein rings of embedding codimension three, Nagoya Math. J. 50 (1973), 227-232.

25. K. Watanabe, Some examples of one dimensional Gorenstein domains, Nagoya Math. J. 49 (1973), 101-109.

Department of Mathematics, University of Kansas, Lawrence, Kansas 66045

Department of Mathematics, University of Tennessee, Knoxville, Tennessee 37916 